1 Bilateral cervical impairment in patients with unilateral lateral epicondylalgia without concomitant cervical or upper limb symptoms: A cross-sectional case control study.

\title{
INTRODUCTION
}

5 Lateral epicondylalgia (LE), also known as tennis elbow, is empirically considered a

6 tendinopathy of the extensor carpi radialis brevis origin. However, there is also clinical

7 recognition of a relationship between the cervical spine, radial nerve and LE. There are

8 findings of a greater prevalence of self-reported neck pain and cervicothoracic impairments in

9 patients with lateral elbow pain compared to an age-matched control population (Berglund et

10 al., 2008) as well as greater radial nerve mechanosensitivity of the affected arm in patients with unilateral symptoms (Yaxley et al., 1993, Wright et al., 1994). Poorer long term prognosis is predicted by self-reported neck pain, independent of high baseline pain intensity (Smidt et al., 2006). Juxtaposed upon this is evidence of the effectiveness of manual therapy directed toward the cervicothoracic spine in conjunction with elbow treatment, including benefit in previously recalcitrant cases (Gunn et al., 1976), fewer treatment sessions (Cleland et al., 2004) and greater improvement in short-term pain (Cleland et al., 2005). Interpretation of the foregoing studies in a clinical context is difficult because it is often not clear whether subjects were excluded if they had neck and upper limb symptoms additional to their LE pain.

This study aimed to evaluate whether there are differences in manual examination of the cervical and thoracic spine between healthy controls and LE subjects who did not have additional (or concomitant) neck or upper limb symptoms. Secondly, the relationship between spinal manual examination and radial nerve neurodynamic test responses was examined in LE subjects, as well as the potential influence of pain severity, duration of injury, age and gender.

$\underline{\text { Design }}$

This comparative study investigated the prevalence of abnormal findings from a clinical examination of the cervical and thoracic spine and radial nerve in individuals with and without unilateral LE. Data for the LE group was collected prior to enrolment into a randomised controlled trial, the methodology for which is described in detail elsewhere (Coombes et al., 2009).

$\underline{\text { Setting and subjects }}$

All subjects were recruited from the greater Brisbane region of Australia through community media advertisements. Eligibility was determined by a two stage process (telephone interview and physical examination) by one researcher (BKC) and confirmed by a second researcher (BV). Criteria for being included in the LE group was unilateral elbow pain over the lateral epicondyle for longer than six weeks and aggravated by a combination of palpation, gripping and resisted wrist and/or finger extension. Exclusion criteria were: recent injection or physiotherapy; exacerbation of elbow pain with neck examination; sensory disturbance of the hands; fractures; elbow surgery; malignancy or inflammatory disorders; pregnancy; breastfeeding; or contraindication to injection. Healthy control subjects aged 35 to 70 with no history of LE were included. All subjects were excluded if they experienced neck or other 
upper limb symptoms necessitating treatment or preventing participation in usual work or recreational activities in the preceding six months. Ethical approval was granted by the institutional review board (University of Queensland) and informed written consent obtained from all subjects.

\section{$\underline{\text { Measures }}$}

The tests used in this study were selected because of their frequent use in clinical assessment of the neck and upper limb. All testing was completed by a single physiotherapist with a postgraduate degree in musculoskeletal physiotherapy who was not blinded to whether the subject had LE or not. Spinal manual examination was performed on all subjects, while neurodynamic function was evaluated in LE subjects only. Subjects were asked to rate the level of elbow pain currently experienced at rest and the worst level of pain experienced during the past week on $100 \mathrm{~mm}$ visual analogue scales (VAS) with the following endpoints: no pain $(0 \mathrm{~mm})$ and worst pain imaginable pain $(100 \mathrm{~mm})$.

\section{Manual examination}

Manual examination of the cervical and thoracic spine between C4 and T2 segments was performed bilaterally on all subjects in prone lying. The examiner rated the mobility at each site on a previously defined scale (Jull et al., 1994), ranging from 1 (severe hypomobility) to 7 (severe hypermobility), with 4 representing normal mobility. The participant verbally rated any pain provoked by examination of each site on an 11-point numerical rating scale. A positive response was defined if moderate to severe hypomobility or hypermobility was present along with a pain response of three or greater (Zito et al., 2006). We based the criteria for impairment on assessment of the quality and range of segmental motion as well as provocation of pain, due to the qualitative nature of assessment of joint motion alone (Jull et al., 1994, Hollerwoger, 2006, Jull et al., 1988). Responses at each site were scored and an aggregate score, consisting of the sum of positive palpation sites, was then derived for further analyses.

\section{Neurodynamic examination}

The upper limb neurodynamic test (ULNT) for the radial nerve was performed as previously described (Butler, 2000), using the following sequencing: shoulder girdle depression, elbow extension, shoulder internal rotation, pronation, wrist and finger flexion and shoulder abduction to the end of range or until symptoms were produced. Once such a sensation was provoked, structural differentiation between neurogenic and non-neurogenic sources of pain was performed by the addition of sensitising movements at a site distant to the pain (shoulder girdle elevation or cervical lateral flexion) while all other test components were maintained. Based on a previous study evaluating the validity of the ULNT (Nee et al., 2012), the test was considered positive if the following two criteria were present: (1) the subject's symptoms were reproduced at least partially; (2) symptoms were altered by structural differentiation. Moderate reliability (Kappa 0.44) has been reported for the radial nerve ULNT using the above criteria (Schmid et al., 2009).

\section{Data management and analysis}

Statistical analysis was performed using SPSS 20 (IBM, Somers, New York, USA), with a $\mathrm{P}<0.05$ significance level. Manual examination of the cervical and thoracic spine between LE and control groups was compared using repeated measures analysis of variance, including the within-subject factors of side (affected or unaffected) and level (C4-5, C5-6, C6-7, C7-T1 or T1-2) and the covariates age and sex. Control subjects were randomly allocated a 
"matched affected arm" such that the control group had an equivalent proportion of dominant sided arms as that observed in the LE group to account for any potential influence of hand dominance (Coombes et al., 2012, Friedman, 1998). Linear and logistic regression models evaluated the relationship between the aggregate score of spinal palpation sites and the ULNT and potential associated factors of severity of pain and disability, age, sex and duration. All variables were simultaneously entered into the model.

\section{$\underline{\text { RESULTS }}$ \\ Analysis was possible using data from 164 subjects with LE and 62 controls without LE (Table 1). Demographic characteristics (Table 1) including age, sex, body mass index, manual occupation and sporting participation were not significantly different between groups. LE subjects had an average ( \pm standard deviation) duration of injury of $25.1 \pm 29.8$ weeks (range six to 25weeks), with worst pain over the previous week and current resting pain levels (VAS) of $61.9 \pm 18.4 \mathrm{~mm}$ and $10.8 \pm 13.4 \mathrm{~mm}$ respectively. This was the first episode of LE in $76.4 \%$ of subjects. Putative causes included work (20.0\%), sport (24.2\%), overload due to unusual activities (23.6\%) and insidious onset (27.3\%). The dominant arm was affected in $71 \%$ of subjects.}

Spinal manual examination responses for LE and control groups at each site are presented in Table 2. Positive responses were most prevalent in LE subjects at C5-6 (18.9\%) and C6-7 (17.7\%) on the same side as their LE. Comparison of LE and control groups using repeated measures analysis of variance found significant group by level $(\mathrm{P}=0.02)$ and group by side $(\mathrm{P}=0.04)$ interactions, in the absence of a three-way interaction. Post hoc investigation of the group by level interaction (Figure 1 ) confirmed that positive tests were significantly more common in LE than control subjects at C4-5 (P=0.01), C5-6 $(\mathrm{P}=0.002)$ and C6-7 $(\mathrm{P}=0.001)$, but not at $\mathrm{C} 7-\mathrm{T} 1$ or $\mathrm{T} 1-2$ levels $(\mathrm{P}>0.05)$. Post hoc investigation of the group by side interaction (Figure 2) showed that positive tests were significantly more common in LE than control subjects, at both the ipsilateral $(\mathrm{P}=0.001)$ and contralateral side $(\mathrm{P}=0.02)$ to the injury.

Evaluation of the number of positive palpation sites showed 36\% of LE subjects had impairment of at least one spinal palpation site, with one subject showing impairment at all sites. Linear regression analysis revealed duration of injury was a significant predictor (Standardised $\beta 0.17,95 \%$ CI 0.001 to $0.18 ; \mathrm{P}=0.03$ ), whereas pain levels, age or sex were not associated with the total number of positive palpation sites. Subjects with more chronic symptoms showed impairment at a greater number of sites than those with more acute symptoms.

A positive ULNT was found in $41 \%$ of LE subjects. Logistic regression found severity of elbow pain experienced at rest (OR 1.03, 95\% CI 1.001 to 1.06; $\mathrm{P}=0.04$ ) and the number of positive palpation sites (OR 1.25, 95\% CI 1.01 to 1.55; $\mathrm{P}=0.04$ ) were significant predictors, whereas worst pain level, duration, age and sex were not associated with neurodynamic response. Subjects with higher resting elbow pain and impairment at multiple cervical or thoracic spinal levels were more likely to have a positive ULNT. 
141 The results of this study indicate that cervical spine impairment, as determined by positive 142 findings on manual palpation, exists in LE subjects who do not have additional neck or upper limb pain. Impairment was evident bilaterally at the cervical spine in unilateral LE, and localised to the lower cervical spine (C4-7) but not the thoracic spine. A longer duration of LE symptoms was associated with a greater risk of cervical spine impairment. Radial nerve mechanosensitivity, as defined as reproduction of LE symptoms during ULNT and changed by sensitisation manoeuvre, was associated with higher severity of elbow pain at rest and more widespread cervical impairment, as inferred by a greater number of positive sites of palpation.

150 With estimates ranging between 57 and 90\% (Berglund et al., 2008, Waugh et al., 2004, the LE population has not been conclusively established. Differences in eligibility criteria, examination procedure and criteria used to detect impairment provide strong sources of heterogeneity between studies. Moreover, the presence of cervical impairment may be seen as a differential diagnosis from true LE (Vicenzino et al., 1996) or as a sub-group thereof (Figure 3). Within our LE population with mean duration of 25 weeks, a subgroup (36\%) of patients displayed impairment of at least one spinal palpation site or had a positive ULNT (41\%). Significantly larger rates were found by Berglund (2008) in their study of 31 patients with lateral elbow pain (Berglund et al., 2008). A majority of subjects (70\%) indicated pain in the cervical or thoracic region on a pain drawing and 55\% had pain (either locally or referred to the elbow) on compression of the cervical vertebral foramina. Positive radial nerve neurodynamic tests were found in $58 \%$ of patients, defined as pain in the forearm at less than 40 degrees of shoulder abduction. These differences may be explained by their more chronic population (mean 36 months) or broader definition of lateral elbow pain, which included cases in which elbow pain was reproduced by cervical examination, arguably a differential diagnosis for LE. In another study, Waugh (2004) investigated 81 patients with unilateral LE (mean duration 31 weeks), excluding those with concurrent upper quadrant symptoms not directly related to their LE (Waugh et al., 2004). Symptomatic cervical signs, defined as at least one active and passive accessory movement (C4-T1) provoking pain and displaying abnormal end-feel, were found in $56 \%$ of cases. Consistent with results of our study, $41 \%$ had a positive ULNT that reproduced their LE symptoms. In a novel study, Pienimaki (2011) recruited 190 patients with unilateral medial or lateral epicondylitis, without exclusion (Pienimaki et al., 2011). On pain drawing, 45\% reported widespread pain over the neck or upper limb, in addition to local elbow and/or forearm pain. Interestingly, widespread pain was also relatively common (39\%) in the subgroup without other diagnosed musculoskeletal disorders (Pienimaki et al., 2011). In this population with chronic symptoms (mean duration 45 weeks), widespread pain was associated with female sex, long duration of symptoms, high pain scores, sick leave and low levels of physical activity.

Our findings of cervical impairment in a subgroup of LE patients without concomitant neck or other arm conditions, provides strength to the growing body of evidence inferring central sensitisation mechanisms (Coombes et al., 2012, June 13, Lim et al., In press, FernandezCarnero et al., 2009, Slater et al., 2005), whereby repeated nociceptor inputs from elbow structures may trigger an increase in the excitability and synaptic efficacy of neurons in central nociceptive pathways (Woolf, 2010). Convergence of afferent input from the lateral elbow and C4-7 cervical segments may underlie the greater incidence of impairment at these spinal segments, whilst receptive field expansion may explain the presence of bilateral impairment in a unilateral condition. We propose that radiculopathy or somatic referral from 
cervical structures is a less likely mechanism underlying findings in our population, due to careful history taking and physical examination, although sub-clinical cases of referred pain cannot be discounted. Another possible mechanism might be related to the generalised motor impairment of the upper limb (Coombes et al., 2012, Alizadehkhaiyat et al., 2007, Bisset et al., 2006) through which altered mechanical loading of the neck during upper limb activities could plausibly promote the development of cervical impairment. Whilst causation cannot be inferred from our cross-sectional study and the size of the associations detected on regression analyses were small, the data lends some support to the notion that more widespread cervical impairment is associated with chronicity. A weak but significant correlation was found between cervical and neurodynamic assessments, indicating that whilst related they may reflect different underlying mechanisms. Positive ULNT was similar in both acute and chronic LE, but was associated with greater resting pain levels. Previous study of chronic LE, did not find an association between positive ULNT and central hyperexcitability as measured by nociceptive withdrawal reflex (Lim et al., In press). It is possible that radial nerve mechanosensitivity represents a normal physiological response to greater pain severity at the time of testing rather than one of augmented central hypersensitivity.

Before drawing clinical implications from this study, it is important to consider several points. Firstly, the population studied was self-referred via community media announcements as a part of a randomised controlled trial and underwent a thorough interview and examination. The examination sought to exclude other comorbid upper limb or cervical symptoms in the experimental sample. This needs to be considered in translation of the findings into clinical practice, that is, the findings relate to a reasonably localised lateral elbow and dorsal forearm pain state. Previous comparison of two LE populations, one of which was recruited in a similar manner to ours, the other recruited from general practice, found similar pain severity, age and history of elbow symptoms between the trials, supporting the generalizability of findings (Bisset et al., 2007).

Secondly, we chose measures that are commonly used in clinical assessment of the upper quarter. However, more confidence in the utility, relevance and importance of these techniques would have been achieved if their reliability had been established in this population and the examination was performed by an investigator blinded to the group or side studied. A previous systematic review questioned the reliability of manual tests alone to detect cervical spine dysfunction (Hollerwoger, 2006). However, the majority of studies included in this review examined either segmental mobility or pain as an outcome. In comparison, our study, along with that by Waugh (2004), defined spinal segments as impaired if they exhibited abnormal motion and provoked pain (Waugh et al., 2004). In addition, we examined multiple segmental levels, in an effort to highlight both the segmental location and extent of spinal impairment. Secondly, while there is insufficient evidence in the literature to support an isolated test of the radial nerve, we aimed to identify patients in whom LE symptoms were at least partly related to the nerves in the neck and arm that had become sensitive to movement. We defined a positive ULNT as reproduction of a patient's LE symptoms and changed by structural differentiation (sensitisation manoeuvres), as recommended in a recent review of the validity of ULNT (Nee et al 2012), consequently the test was not performed in control participants as they did not experience such symptoms. Previous studies have found a high rate of false positive tests in asymptomatic individuals and recommended that reproduction of the patient's symptoms should be an integral part of the diagnostic criteria (Davis et al., 2008).

This cross-sectional study of 222 subjects provides a valuable summary of spinal and neurodynamic function and might assist in clinical decision making regarding use of physical 
modalities for LE. Preliminary work has demonstrated the effectiveness of manual therapy directed to the cervical spine in conjunction with elbow treatment for patients with LE exhibiting cervical impairment (Cleland et al., 2005). Our results highlight patients with longer duration of LE as potential candidates for spinal manual therapy and may explain the greater hypoalgesic effect of cervical compared to thoracic spinal manipulation in this population (Fernandez-Carnero et al., 2011). Secondly, results suggest that techniques to reduce nerve mechanosensitivity may be of benefit in a subgroup of patients with more severe resting pain. Whilst the effectiveness of neural tissue management has not been addressed in LE, immediately clinically relevant benefits have been demonstrated in patients with nerve-related neck and arm pain, using cervical manual therapy and a home exercise program of nerve gliding exercises (Nee et al., 2012).

247 Differentiation of LE subgroups with cervical and neural impairment might be an important 248 prerequisite for effective treatment, and evaluation of their prognostic capacity is necessary. 249 It is important that future efforts are made to improve the reliability of measures of cervical 250 and neurodynamic impairment so that subgroups can be determined with improved veracity. 251 Careful exclusion of patients with referred pain or other concomitant neck or upper limb conditions by this study provides a logical step for this field of interest. 
257 1. Berglund KM, Persson BH, Denison E. Prevalence of pain and dysfunction in the cervical and thoracic spine in persons with and without lateral elbow pain. Man Ther. 2008;13:295-9. 2. Yaxley GA, Jull GA. Adverse tension in the neural system. A preliminary study of tennis elbow. Aust J Physiother. 1993;39(1):15-22.

3. Wright A, Thurnwald P, O'Callahan J, Smith J, Vicenzino B. Hyperalgesia in tennis elbow patients. J Musculoskel Pain. 1994;2:83097.

4. Smidt N, Lewis M, Van Der Windt D, Hay EM, Bouter LM, Croft P. Lateral epicondylitis in general practice: course and prognostic indicators of outcome. J Rheumatol. 2006;33(10):2053-59.

5. Gunn CC, Milbrandt WE. Tennis elbow and the cervical spine. Can Med Assoc J. 1976;114(9):803-9.

6. Cleland JA, Whitman JM, Fritz JM. Effectiveness of manual physical therapy to the cervical spine in the management of lateral epicondylalgia: a retrospective analysis. J Orthop Sports Phys Ther. 2004;34(11):713-22; discussion 22-4.

7. Cleland J, Flynn TW, Palmer JA. Incorporation of manual therapy directed at the cerviothoracic spine in patients with lateral epicondylalgia. A pilot clinical trial. J Man Manip Ther. 2005;13(3):143-51.

8. Coombes BK, Bisset L, Connelly LB, Brooks P, Vicenzino B. Optimising corticosteroid injection for lateral epicondylalgia with the addition of physiotherapy: a protocol for a randomised control trial with placebo comparison. BMC Musculoskelet Disord. 2009;10:76.

9. Jull G, Treleaven J, Versace G. Manual examination: is pain provocation a major cue for spinal dysfunction? Aust J Physiother. 1994;40:159-65.

10. Zito G, Jull G, Story I. Clinical tests of musculoskeletal dysfunction in the diagnosis of cervicogenic headache. Man Ther. 2006;11(2):118-29.

11. Hollerwoger D. Methodological quality and outcomes of studies addressing manual cervical spine examinations: a review. Man Ther. 2006;11(2):93-8.

12. Jull G, Bogduk N, Marsland A. The accuracy of manual diagnosis for cervical zygapophysial joint pain syndromes. Med J Aust. 1988;148(5):233-6.

13. Butler D. The Sensitive Nervous System. Unley: NOI Group Publications; 2000.

14. Nee RJ, Jull G, Vicenzino B, Coppieters M. The Validity of Upper-Limb Neurodynamic Tests for Detecting peripheral Neuropathic Pain. JOSPT. 2012;42(5):413-24.

15. Schmid AB, Brunner F, Luomajoki H, Held U, Bachmann LM, Kunzer S, et al. Reliability of clinical tests to evaluate nerve function and mechanosensitivity of the upper limb peripheral nervous system. BMC Musculoskelet Disord. 2009;10:11.

16. Coombes BK, Bisset L, Vicenzino B. Elbow flexor and extensor muscle weakness in lateral epicondylalgia. Br J Sports Med. 2012;46(6):449-53.

17. Friedman PJ. Isokinetic peak torque in women with unilateral cumulative trauma disorders and healthy control subjects. Arch Phys Med Rehabil. 1998;79(7):816-9.

18. Waugh EJ, Jaglal SB, Davis AM, Tomlinson G, Verrier MC. Factors associated with prognosis of lateral epicondylitis after 8 weeks of physical therapy. Arch Phys Med Rehabil. 2004;85(2):308-18. 19. Vicenzino $B$, Collins $D$, Wright $A$. The initial effects of a cervical spine manipulative physiotherapy treatment on the pain and dysfunction of lateral epicondylalgia. Pain. 1996;68(1):6974.

20. Vicenzino B, Wright A. Lateral epicondylalgia 1:Epidemiology, pathophysiology, aetiology and natural history. Physical Therapy Reivew. 1996;1:23-4.

21. Pienimaki T, Siira P, Vanharanta H. Widespread pain in chronic epicondylitis. Eur J Pain. 2011;15(9):921-7.

22. Coombes BK, Bisset L, Vicenzino B. Thermal hyperalgesia distinguishes those with severe pain and disability in unilateral lateral epicondylalgia. Clin J Pain. 2012, June 13;Epub. 

hyperexcitability as measured with nociceptive flexion reflex (NFR) threshold in chronic lateral epicondylalgia with or without positive neurodynamic test. J Pain. In press. Nielsen L. Widespread mechanical pain hypersensitivity as sign of central sensitization in unilateral epicondylalgia: a blinded, controlled study. Clin J Pain. 2009;25(7):555-61. 25. Slater H, Arendt-Nielsen L, Wright A, Graven-Nielsen T. Sensory and motor effects of experimental muscle pain in patients with lateral epicondylalgia and controls with delayed onset muscle soreness. Pain. 2005;114(1-2):118-30. 26. Woolf CJ. Central sensitization: implications for the diagnosis and treatment of pain. Pain. 2010;152(3 Suppl):S2-15. imbalance in tennis elbow: a functional and electromyographic assessment. J Orthop Res. 2007;25(12):1651-7.

319 28. Bisset LM, Russell T, Bradley S, Ha B, Vicenzino BT. Bilateral sensorimotor abnormalities in unilateral lateral epicondylalgia. Arch Phys Med Rehabil. 2006;87(4):490-5. treatments for tennis elbow do subgroups of patients respond differently? Rheumatology (Oxford). 2007;46(10):1601-5.

30. Davis DS, Anderson IB, Carson MG, Elkins CL, Stuckey LB. Upper Limb Neural Tension and Seated Slump Tests: The False Positive Rate among Healthy Young Adults without Cervical or Lumbar Symptoms. J Man Manip Ther. 2008;16(3):136-41.

31. Fernandez-Carnero J, Cleland JA, Arbizu RL. Examination of motor and hypoalgesic effects of cervical vs thoracic spine manipulation in patients with lateral epicondylalgia: a clinical trial. J Manipulative Physiol Ther. 2011;34(7):432-40. immediate clinically relevant benefits without harmful effects for patients with nerve-related neck and arm pain: a randomised trial. Journal of physiotherapy. 2012;58(1):23-31. 
Figure 1. Results of spinal manual examination in lateral epicondylalgia (LE) and control (C) subjects at each segmental level. Data illustrates the significant group by level interaction.

Figure 2. Results of spinal manual examination in lateral epicondylalgia (LE) and control (C) subjects at the side ipsilateral and contralateral to injury. Data illustrates the significant group by side interaction.

Figure 3. Cervical impairment may exist in a subgroup of patients with lateral epicondylalgia 346 or implicate a differential diagnosis. 